\title{
Detailed structures of accretion and outflow probed by molecular masers in high-mass protostars
}

\section{Ciriaco Goddi*}

Joint Institute for VLBI in Europe, Postbus 2, NL-7990 AA Dwingeloo, the Netherlands

E-mail: goddi@jive.nl

Luca Moscadelli

INAF, Osservatorio Astrofisico di Arcetri, Largo E. Fermi 5, 50125 Firenze, Italy

E-mail: mosca@arcetri.astro.it

\section{Alberto Sanna}

Max-Planck-Institut für Radioastronomie, Auf dem Hügel 69, 53121 Bonn, Germany

E-mail: asanna@mpifr-bonn.mpg.de

Multi-epoch VLBI observations of intense molecular masers have been proved to be a unique tool to study the 3D dynamics of circumstellar gas at distances of tens/hundreds of AU from massive young stellar objects (YSOs). Specifically, water masers typically trace shocked material in protostellar winds/jets; Class II methanol masers may probe accreting/infalling structures in disks/envelopes. Based on a small sample of high-mass YSOs, we identified two different morphologies of outflows and two phases of accretion. I) In a B-type YSO (probably in pre-ZAMS), a shock-excited radio jet is responsible for the excitation of water masers in a "collimated bipolar flow"; an infalling envelope probed by methanol masers indicates that accretion is still ongoing onto the central YSO. II) In an O-type YSO (probably already in the ZAMS), a HC-HII region expands (likely driven by a stellar wind), excites and accelerates the water masers in a "wideangle shell"; a surrounding toroid traced by methanol masers (and thermal lines) contains gas that is infalling and rotating on large-scales, but its accretion may be halted at the shock front where water masers are excited, indicating that the YSO may have reached its final mass. We propose that these two different morphologies of outflows and two phases of accretion may reflect an evolutionary effect and/or a trend with protostellar mass. An extension of this study to a statistically significant sample will enable us to assess if these different phases of protostellar ejection and accretion observed in our small sample are a common pattern in the evolution of young massive stars.

11th European VLBI Network Symposium \& Users Meeting,

October 9-12, 2012

Bordeaux, France

\footnotetext{
*Speaker.
} 


\section{Introduction}

In the formation process of low-mass stars, disks and jets/outflows play a major role controlling the accretion of matter onto the forming star (disks) and removing the excess of angular momentum (jets). The formation of massive stars by the same accretion processes believed to form low-mass stars appears problematic, because the intense radiation pressure from the star luminosity and the thermal pressure from the H II region around the young stellar objects (YSOs) may be sufficient to reverse the accretion flow and prevent matter from reaching the star. In addition, while lowmass protostellar jets are expected to be launched and collimated via magneto-centrifugal processes within a narrow opening angle $\left(\leq 10^{\circ}\right)$ at distances $<10 \mathrm{AU}$ from the star [1], for massive YSOs the ionization feedback and the dynamical effects of the stellar radiation on the surrounding gas could cause a significant decollimation of protostellar outflows [2]. These theoretical considerations appear to agree with the results of an observational survey of massive molecular outflows based on interferometric imaging of $\mathrm{mm}$ lines from several outflow tracers, which seems to suggest that early B-type YSOs show on average more collimated outflows than late O-type YSOs [3]. However, high extinctions, clustering, and large distances (typically several kpc) of massive star forming regions (SFRs) hinder attempts to resolve the structure and dynamics of outflowing gas at small radii ( $\lesssim 1000 \mathrm{AU}$ ) from YSOs (present mm interferometers have typical resolutions of order $1^{\prime \prime}$ ). In this context, one excellent diagnostic tool of gas kinematics within 10-100 AU from YSOs is provided by VLBI observations of interstellar masers [4, 5, 6, 7, 8, 9, 10, 11]. For over a decade, we have been conducting an observational campaign towards a sample of massive YSOs $(\sim 10)$, complementing VLBI monitoring of masers with interferometric observations of thermal tracers.

Here, we report results from a detailed study towards two SFRs, representing two classes of objects in our sample: I) AFGL $5142(1.8 \mathrm{kpc})$ is an intermediate-mass SFR with a moderate bolometric luminosity $\left(\sim 5 \times 10^{3} L_{\odot}\right)$ where a cluster of young stars is forming, containing Btype objects; II) G24.78+0.08 (7.7 kpc) is a high-mass SFR with a higher bolometric luminosity $\left(8 \times 10^{4} L_{\odot}\right)$ where a cluster of young stars including O-type objects is forming.

These studies show that physical and dynamical properties of accretion and outflow differ in these two types of objects. This may reflect general properties intrinsic to the formation and evolution of massive stars.

\section{Observations and Results}

In both targets, we observed $6.7 \mathrm{GHz} \mathrm{CH} \mathrm{CH}_{3} \mathrm{OH}$ masers with the $\mathrm{EVN}$ at three distinct epochs (spanning 5 years), $22.2 \mathrm{GHz} \mathrm{H}_{2} \mathrm{O}$ masers with the VLBA at four distinct epochs (spanning a few months), and radio continuum emission with the VLA at 8.4, 22, and $43 \mathrm{GHz}$.

AFGL 5142. Observations with the SMA at $1.3 \mathrm{~mm}$ identified a high-mass protocluster containing five dusty $\mathrm{mm}$ cores and three CO outflows, within a few arcseconds [12]. The focus here is the mm core MM-1, which shows hot-core chemistry, exhibits radio continuum emission from ionized gas, and powers strong water and methanol masers [6]. The 3D kinematics of $\mathrm{H}_{2} \mathrm{O}$ and $\mathrm{CH}_{3} \mathrm{OH}$ masers is discussed in detail in [7]. Figure 1 shows the proper motions of $\mathrm{H}_{2} \mathrm{O}$ masers (left panel) and $\mathrm{CH}_{3} \mathrm{OH}$ masers (right panel), overplotted on the contour maps of the continuum emission at $8.4 \mathrm{GHz}$ and $22 \mathrm{GHz}$. The proper motions should represent the sky-projected ve- 
locities as measured by an observer comoving with the star (see [7] for details). The $22 \mathrm{GHz}$ continuum emission ( 0. '24 beamwidth) appears elongated NW-SE, while the $8.4 \mathrm{GHz}$ emission ( 0 .' 16 beamwidth) is resolved in two components separated by 300 mas (or 540 AU) on the plane of the sky. $\mathrm{H}_{2} \mathrm{O}$ masers are concentrated in two clusters, associated with the two components of the 8.4 GHz continuum, one with blue-shifted line-of-sight velocities (SE) and one with red-shifted line-of-sight velocities (NW). Based on their proper motions, both clusters are moving away from each other along NW-SE, with velocities $\sim 15 \mathrm{~km} \mathrm{~s}^{-1}$. Based on our measurements of positions and $3 \mathrm{D}$ velocities of water masers and under reasonable assumptions for gas densities, we can derive geometrical and mechanical parameters of the outflow: a semi-opening angle of $30^{\circ}$, a mass-loss rate $\dot{M}_{\text {out }}=1.6 \times 10^{-4} n_{8} M_{\odot} \mathrm{yr}^{-1}$ and a momentum-rate $\dot{P}_{\text {out }}=2.4 \times 10^{-3} n_{8} M_{\odot} \mathrm{yr}^{-1}$ $\mathrm{km} \mathrm{s}^{-1}$, where $n_{8}$ is the ambient volume density in units of $10^{8} \mathrm{~cm}^{-3}$ (required for water maser excitation; e.g., [13]). The relatively high mass-loss and momentum rates indicate strong outflow activity from MM-1. Interestingly, [12] identified a $\mathrm{CO}$ outflow extended over $0.5 \mathrm{pc}$ and oriented NW-SE, with a position angle similar to the water maser flow. They derived a momentum rate of $2 \times 10^{-3} M_{\odot} \mathrm{yr}^{-1} \mathrm{~km} \mathrm{~s}^{-1}$, which is in good agreement with the momentum rate of the $\mathrm{H}_{2} \mathrm{O}$ maser outflow. We conclude that the ionized jet observed in the radio continuum emission and the molecular outflow probed by water masers, can account for the acceleration of the large-scale CO outflow driven by MM-1.

$\mathrm{CH}_{3} \mathrm{OH}$ masers are distributed across a similar area as the $\mathrm{H}_{2} \mathrm{O}$ masers, and consist mainly of two clusters: a cluster with moderately red-shifted line-of-sight velocities ("yellow") is associated with the NW continuum peak; another cluster with the most red-shifted line-of-sight velocities ("red") lies in between the two $8.4 \mathrm{GHz}$ peaks (Figure 1, right panel). The red masers are projected in the plane of the sky closer to the protostellar position and move mostly along the line-of-sight, whereas the yellow masers have proper motions with larger amplitudes and directed towards the red maser centroid; both aspects indicate infall towards the protostar. This is consistent with methanol masers having red-shifted line-of-sight velocities and being in the foreground of the $22 \mathrm{GHz}$ continuum emission (optically thick at $6.7 \mathrm{GHz}$ ). We can use a simple spherical model for the infall, to estimate the infalling envelope radius $(R=290 \mathrm{AU})$ and mass $\left(4 M_{\odot}\right)$, the infall velocity $\left(V_{\text {inf }}=5 \mathrm{~km} \mathrm{~s}^{-1}\right)$, and the mass infall rate $\left(\dot{M}_{\text {inf }}=6 \times 10^{-4} n_{8} M_{\odot} \mathrm{yr}^{-1}\right)$. The high infall rate indicates that the protostar is actively accreting.

In summary, the knowledge of the 3D velocity field of molecular gas in AFGL5142 enabled us to characterize the protostellar outflow structure on scales of few hundreds of AU from the kinematics of $\mathrm{H}_{2} \mathrm{O}$ masers and provided a direct measurement of infall of a circumstellar envelope with a radius of only $300 \mathrm{AU}$ onto an intermediate-mass protostar $\mathrm{CH}_{3} \mathrm{OH}$ masers.

G24.78+0.08. The SFR contains several distinct centers of star formation spread over $\sim 0.4 \mathrm{pc}$, indicated by the presence of different hot (molecular and dusty) cores and HII regions [14], typical of a massive star forming cluster. The description here focus on one specific core, G24 A1, which is a $20 M_{\odot}$ YSO surrounded by a HC-HII region, driving a $\mathrm{CO}$ bipolar outflow, and located at the center of a massive rotating toroid undergoing infall towards the HC-HII region $[15,16,17]$.

The $6.7 \mathrm{GHz}$ maser emission observed with the EVN comes from two clusters: the first one is distributed around the HC-HII region (mapped with the VLA at 7mm, [16]) and has redshifted velocities, the second one with blueshifted velocities is observed towards the SW with respect to 

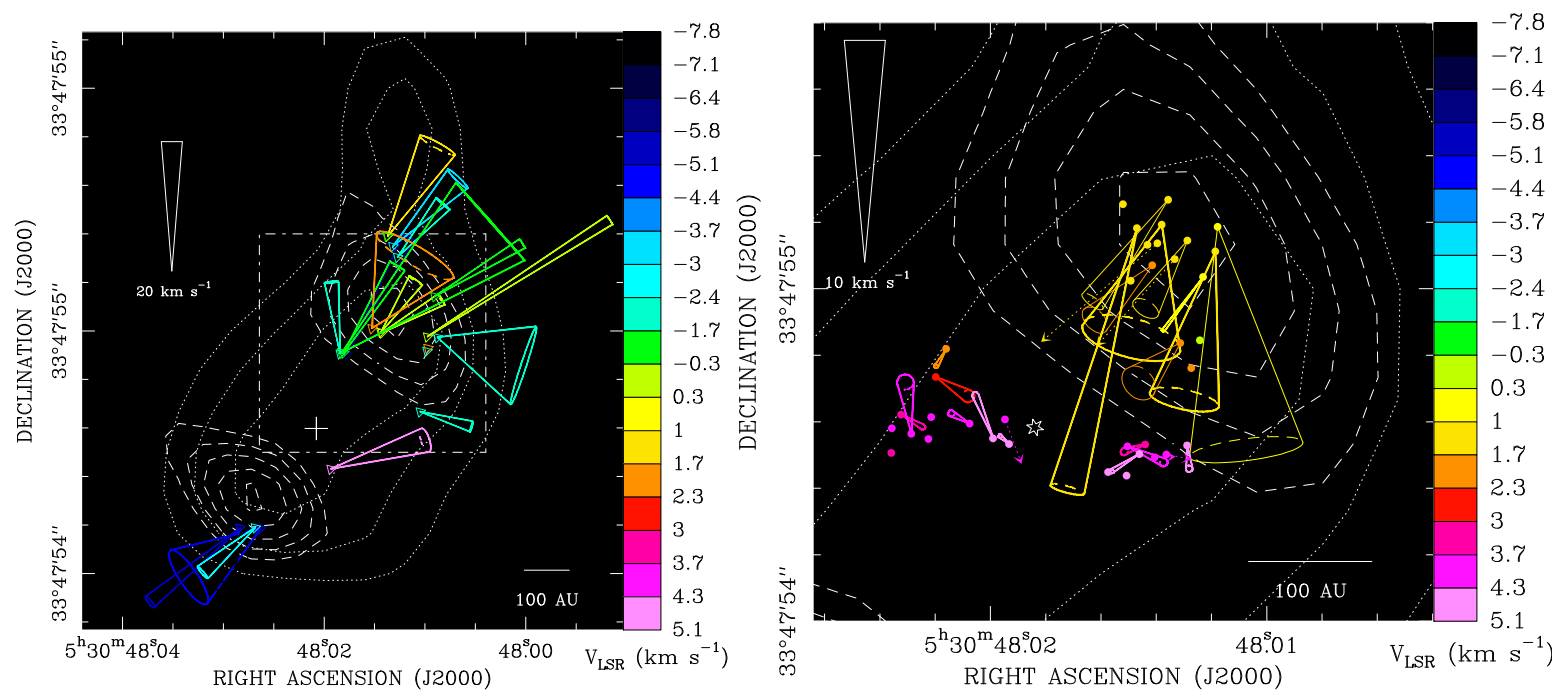

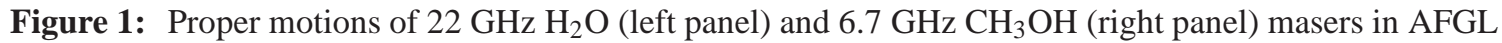
5142 MM-1, observed with the VLBA and the EVN, respectively. The rectangle in the left panel shows the area plotted in the right panel. The cones indicate orientation and uncertainties of measured proper motions (the amplitude scale is given in each panel) and colors denote line-of-sight velocities. Contour maps show the VLA $22 \mathrm{GHz}$ (dotted line) and $8.4 \mathrm{GHz}$ (dashed line) continuum. The white star in the right panel identifies the putative location of the protostar.

the HC-HII region (Figure 2, left panel). Interestingly, a $\mathrm{V}_{\mathrm{LSR}}$ gradient is observed along a NESW direction in the $\mathrm{CH}_{3} \mathrm{CN}$ (12-11) line at $1.4 \mathrm{~mm}$ imaged with the PdBI [15], approximately perpendicular to the axis of a CO molecular outflow (Figure 2, left panel, bottom left inset). The elongated distribution and the line-of-sight velocity variation of methanol masers agree well with the axis of the velocity gradient measured in the $\mathrm{CH}_{3} \mathrm{CN}$ line, indicating that in this source methanol masers can arise from the rotating disk. Assuming that the gas is in rotational equilibrium, we derive a dynamical mass of $20 M_{\odot}$.

Absolute positions and 3D velocities of the water masers trace an arc-shaped structure (with size $\sim 0.01 \mathrm{pc}$ ) at the border of the HC-HII region and are expanding from the position of the YSO, indicated by the peak of the $7 \mathrm{~mm}$ continuum emission (Figure 2, right panel). This finding suggests that the water maser motions are driven by the expansion of the ionized gas. Thermal pressure is excluded because the water maser velocities largely exceed the sound speed of the ionized gas $\left(10 \mathrm{~km} \mathrm{~s}^{-1}\right.$ for gas at $\left.10^{4} \mathrm{~K}\right)$. This indicates that the expansion must be driven by an additional mechanism, in this case we propose a powerful stellar wind emitted by the O-type star ionizing the gas. This finding however appears to contradict the fact that the gas on a larger scale is infalling towards the HC-HII region [17]. The contradiction becomes evident when comparing the structure and velocity fields of methanol and water masers in this source. Methanol masers lie further from the HC-HII region than the water masers and appear to probe molecular gas in the rotating toroid, traced by the $1.4 \mathrm{~mm} \mathrm{CH}_{3} \mathrm{CN}$ thermal line. On the one hand, this suggests that the YSO may be still accreting material and that the HC-HII region may trace the ionized portion of the accretion flow (e.g., [18]). On the other hand, the water masers trace a "wide-angle" flow expanding right from the border of the HC-HII region. A possible reconciling explanation is that the infalling material 

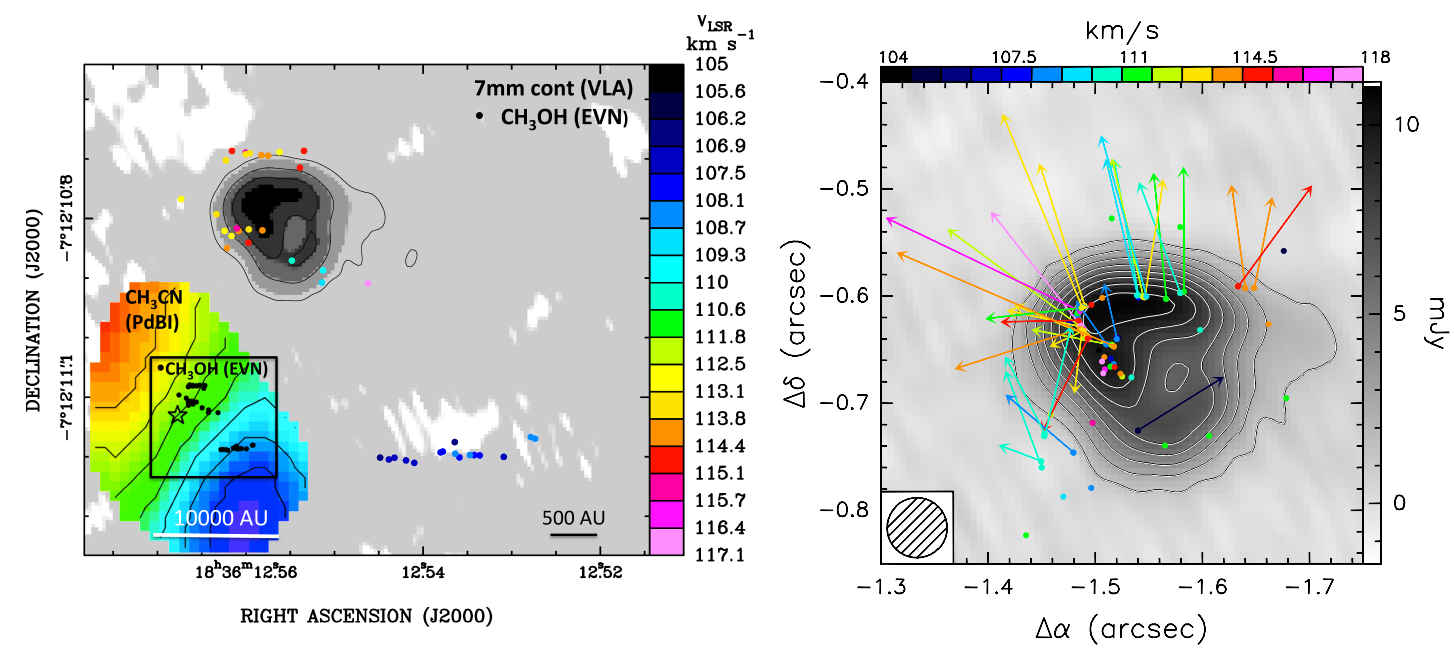

Figure 2: $6.7 \mathrm{GHz}$ methanol masers observed with the EVN (left panel) and $22 \mathrm{GHz}$ water masers observed with the VLBA (right panel) towards the G24 A1 core. Colors code line-of-sight velocities, according to the wedge to the right. The colored arrows indicate the proper motions of $\mathrm{H}_{2} \mathrm{O}$ masers. In both panels, the grey-scale map shows the $7 \mathrm{~mm}$ continuum emission observed with the VLA in A-Array, which traces a HC-HII region with a radius $\sim 600 \mathrm{AU}$. The inset shows a map of the $\mathrm{CH}_{3} \mathrm{CN}$ (12-11) line peak velocity observed with the PdBI [15], overlaid with the positions of $\mathrm{CH}_{3} \mathrm{OH}$ masers (black spheres).

in the toroid can no longer accrete onto the YSO, but is halted at the surface of the HC-HII region, right at the shock front where water masers are excited. In this interpretation, the water maser shell should mark the border of the dynamical interaction between the YSO and the surrounding ambient medium. On the other hand, methanol masers, which are located beyond the border of the HC-HII region, would trace pre-shock ambient gas, unaffected by the expansion of the HII region, and therefore might be still rotating and slightly contracting. If this interpretation is correct, the methanol masers should be participating in the infall and their proper motions should be directed towards the HC-HII region. Further VLBI measurements are however bound to confirm or rule out this scenario.

\section{Discussion and Conclusions}

Our VLBI study of water and methanol masers combined with the morphology of radio continuum (imaged with the VLA) has revealed two different typologies of outflow and accretion: I) In a B-type YSO (probably in pre-ZAMS), a shock-excited radio jet is responsible for the excitation of water masers in a collimated bipolar flow; an infalling envelope probed by methanol masers indicates that accretion is still ongoing onto the central YSO.

II) In an O-type YSO (probably already in the ZAMS), a HC-HII region expands (likely driven by a stellar wind), excites and accelerates the water masers in a wide-angle shell; a surrounding toroid traced by methanol masers (and thermal lines) contains gas that is infalling and rotating on large-scales, but its accretion may be halted at the shock front where water masers are excited. In this case the YSO may have reached its final mass.

In this contribution, we presented just two cases, but similar properties are observed in a larger sample of massive SFRs studied with multi-epoch VLBI observations [7, 8, 10, 11, 19, 20]. 
The wide-angle or collimated flows observed on scales of $\sim 100 \mathrm{AU}$, provide a strong observational evidence of an intrinsic different degree of outflow collimation associated with more massive (O-type vs. B-type) and/or more evolved (ZAMS vs. pre-ZAMS) YSOs. Similarly, the different phases of accretion inferred from methanol measurements may reflect an evolutionary effect and/or a trend with protostellar mass. In order to assess if these different phases of ejection/accretion observed in our small sample are a common pattern in the evolution of young massive stars, we plan to extend our study to a statistically significant sample of massive SFRs. In this context, the "BeSSeL" project [21] provides a larger and well-suited sample of massive SFRs studied with multi-epoch VLBI observations of methanol and water masers. Complementary observations of radio continuum with the JVLA and of hydrogen recombination lines with ALMA as well as observation of thermal lines with both interferometers, will provide the complementary information on the morphology and kinematics of ionized and molecular gas on the relevant scales. This will eventually enable us to study in detail the evolution of outflows and properties of accretion in a large sample of massive SFRs, on scales from tens to thousands of AU.

\section{References}

[1] Pudritz, R. E., Ouyed, R., Fendt, C., \& Brandenburg, A. 2007, Protostars and Planets V, 277

[2] Vaidya, B., Fendt, C., Beuther, H., \& Porth, O. 2011, ApJ, 742, 56

[3] Beuther, H., \& Shepherd, D. 2005, Cores to Clusters: Star Formation with Next Generation Telescopes, 105

[4] Goddi, C., Moscadelli, L., Alef, W., et al. 2005, A\&A, 432, 161

[5] Goddi, C., \& Moscadelli, L. 2006, A\&A, 447, 577

[6] Goddi, C., Moscadelli, L., Sanna, A., Cesaroni, R., \& Minier, V. 2007, A\&A, 461, 1027

[7] Goddi, C., Moscadelli, L., \& Sanna, A. 2011, A\&A (Letters), 535, L8

[8] Moscadelli, L., Goddi, C., Cesaroni, R., Beltrán, M. T., \& Furuya, R. S. 2007, A\&A, 472, 867

[9] Moscadelli, L., Sanna, A., \& Goddi, C. 2011, A\&A, 536, A38

[10] Sanna, A., Moscadelli, L., Cesaroni, R., et al. 2010a, A\&A, 517, A71

[11] Sanna, A., Moscadelli, L., Cesaroni, R., et al. 2010b, A\&A, 517, A78

[12] Zhang, Q., Hunter, T. R., Beuther, H., et al. 2007, ApJ, 658, 1152

[13] Kaufman, M. J., \& Neufeld, D. A. 1996, ApJ, 456, 250

[14] Furuya, R. S., Cesaroni, R., Codella, C., et al. 2002, A\&A, 390, L1

[15] Beltrán, M. T., Cesaroni, R., Neri, R., et al. 2004, ApJ, 601, L187

[16] Beltrán, M. T., Cesaroni, R., Moscadelli, L., \& Codella, C. 2007,A\&A, 471, L13

[17] Beltrán, M. T., Cesaroni, R., Codella, C., et al. 2006, Nature, 443, 427

[18] Galván-Madrid, R., Rodríguez, L. F., Ho, P. T. P., \& Keto, E. 2008, ApJ, 674, L33

[19] Torrelles, J. M., Patel, N. A., Gómez, J. F., et al. 2001, ApJ, 560, 853

[20] Torrelles, J. M., Patel, N. A., Anglada, G., et al. 2003, ApJ, 598, L115

[21] Brunthaler, A., Reid, M. J., Menten, K. M., et al. 2011, Astronomische Nachrichten, 332, 461 